\title{
Failure to find the "local" EEG OR to low-level auditory stimulation
}

\author{
ROBERT J. BARRY \\ School of Education, University of New South Wales, Kensington 2039, Australia
}

\begin{abstract}
Sokolov's dichotomization of the EEG OR into "local" and "general" on the basis of differential habituation rates was investigated with special reference to the auditory EEG OR. The magnitude of alpha desynchronization at stimulus onset was examined in the auditory, visual, and motor projection areas as a function of stimulus intensity and stimulus repetition. Significant intensity and repetition effects were found as required for any $\mathrm{OR}$ indicator, but these did not vary with recording site. Thus no evidence for the existence of a "local" auditory EEG OR was found in the present study.
\end{abstract}

The writings of E. N. Sokolov have generated much experimental activity, during the past decade, aimed at verifying and extending his concept of the orienting response (OR). One aspect of this theory, namely his distinction between local and general EEG ORs, has gone unchallenged in the literature. Although it is treated in important texts (e.g., Grossman, 1967) as a valid distinction, this may be unfortunate since there is some evidence that the concepts are confused, even in standard secondary sources elucidating Sokolov's position. The local/general distinction was probably most succinctly put by Sokolov (1960, pp. 189-190):

"Two different forms of orienting reflex can be distinguished .... When we record simultaneously the occipital EEG, the EEG of the motor region, the skin galvanic response, muscle tension, eye movements, and respiration, the first presentation of tactile stimulation produces a generalized response."

"After twenty-four presentations, the only effect is a slight depression of EEG in the motor region . . . . Therefore, during the course of repeated presentations, the generalized form of the orienting response .... changes into a local form, which is a kind of activation of the specific analyzer that is being stimulated."

From this it appears that the general EEG OR is conceptualized as a widespread desynchronization of the alpha rhythm produced by a variety of stimuli (leading to the property usually described as "non-specificity" [Sokolov, 1963b]). This habituates rapidly with repeated stimulation, leaving a local desynchronization which depends on the modality of the stimulation. Examples of local ORs in the scalp EEG of man are given for occipital and rolandic rhythms (with visual and tactile stimulation, respectively) bj Sokolov (1960, 1961, 1963b), and

Requests for reprints should be addressed to Robert J. Barry, School of Education, University of New South Wales, Kensington 2033, Australia. The author thanks Dr. Helen C. Beh for her comments during the course of this study, and her reading of an earlier draft of this paper. local ORs in the auditory projection area are referred to by Sokolov (1963a, 1968).

It is surprising that this dichotomy of response type has been confused with the tonic/phasic distinction of Sharpless and Jasper (1956), but Lynn (1966), probably the most influential secondary reference on Sokolov's OR theory, claimed an equivalence between these two dichotomies. This confusion probably arose from the fact that Sokolov (1955) reported the existence of tonic and phasic forms of the OR compatible with Sharpless and Jasper (1956) (this was mentioned also in a footnote of Sharpless \& Jasper). These two phenomena must be recognized as different dichotomizations of the EEG index of the OR to simple stimulation.

The only Western work which is of relevance to this question of the local OR in the scalp EEG of man is that of Gastaut, Jus, Jus, Morrell, Storm Van Leeuwen, Dongier, Naquet, Regis, Roger, Bekkering, Kamp, and Werre (1957). While these authors have reported results which are supportive of Sokolov's position in the broad division between general and local EEG responses, there is an important difference in the conceptualization of the "local" desynchronization in the region corresponding to the analyzer in question. Sokolov used the auditory, visual, and motor projection systems as three independent processing units with characteristic local ORs produced by stimulation in the appropriate modality. On the other hand, Gastaut et al. (1957) differentiated these three modalities into only two systems: the rolandic region for somaesthetic stimuli and the temporo-parietooccipital region for visual and auditory stimuli. In a footnote (p. 26), they clarify this distinction: "Nous avons dé jà signalé, en effet, que nous ne sommes parvenus à désynchroniser que la région rolandique en utilisant des stimuli somesthésiques ou la région occipito-parieto-temporale en employant des stimuli visuels ou auditifs (nous ne sommes pas arrivés notemment à bloquer électivement le rythme occipital avec des stimuli visuels ni le rhythme temporal avec 


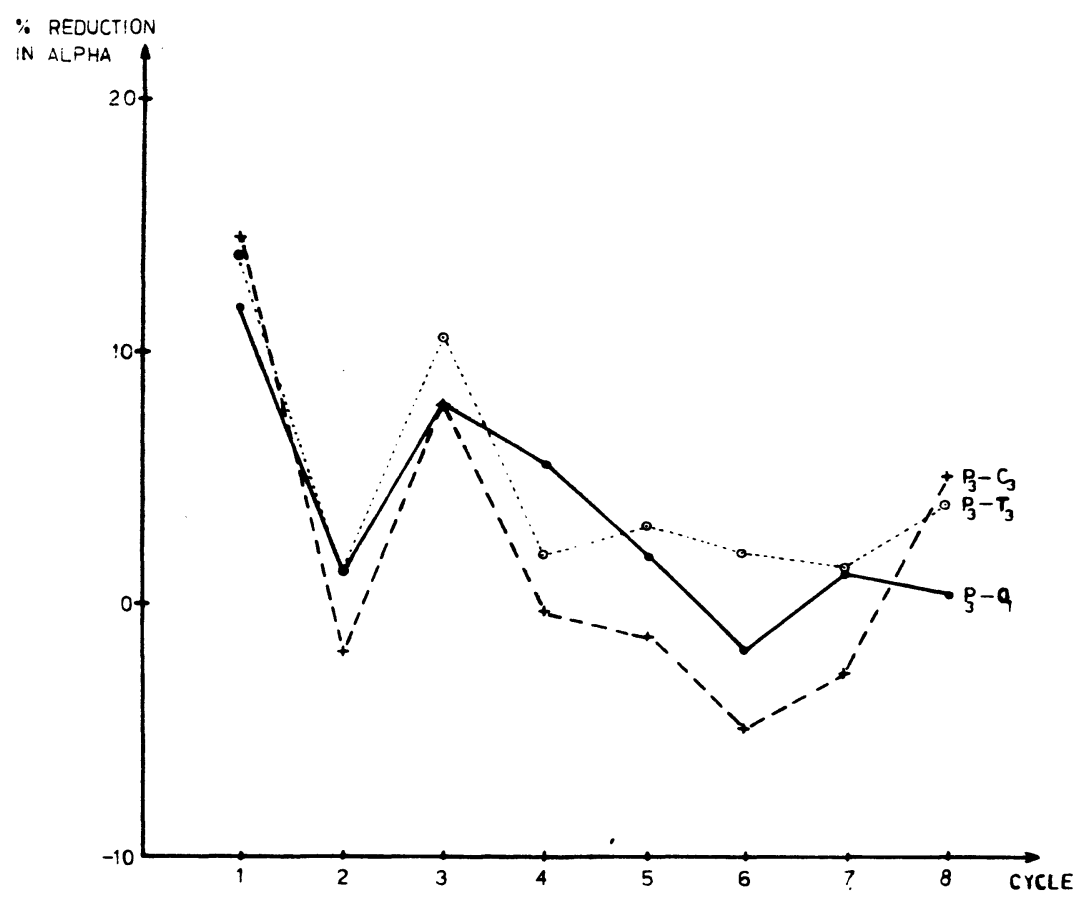

Figure 1. Change in alpha activity in each channel as a function of repetition of the stimulus cycle. des stimuli auditifs, mais seulement le rythme occipito-temporal et même pariétal avec ces deux sortes de stimuli)." This is obviously contrary to the results claimed by Sokolov. It should also be noted that neither Sokolov nor Gastaut et al. (1957) has reported any quantitative measures of EEG activity; their conclusions are based only on visual inspection of individual protocols. These problems have led to the present study aimed at elucidating the nature of this "local" EEG OR.

\section{METHOD}

Subjects were 24 males under 25 years of age who participated to fulfill a requirement of an introductory psychology course. They were seated with eyes open in the dark in a comfortable armchair in an electrically shielded light-tight sound-attenuated chamber. Subjects were fitted with silver/silver chloride pad electrodes at $T_{3}$ (auditory projection area), $\bar{C}_{3}$ (motor projection area), $\mathrm{O}_{1}$ (visual projection area), $P_{3}$, and $C_{z}$, following Jasper (1958). $C_{z}$ was earthed and recording was made on a Grass IIID EEG (with HF cutoff at $50 \mathrm{~Hz}$ ) of the activity between $\mathrm{P}_{3}-\mathrm{T}_{3}, \mathrm{P}_{3}-\mathrm{C}_{3}$, and $\mathrm{P}_{3}-\mathrm{O}_{1}$ to estimate activity in the three projection areas relating to the auditory, tactile, and visual modalities, respectively. Eye movements were also monitored as a possible source of artifact. In addition, the galvanic skin response, heart rate, cephalic and digital pulse volumes, and respiration rate were recorded but are not relevant to the present problem and are not reported here.

Stimuli consisted of $2-\mathrm{sec} 1,000-\mathrm{Hz}$ tones at $20,30,40$, and $50 \mathrm{~dB}$ SPL with an interstimulus interval between 20 and $100 \mathrm{sec}$ (mean $60 \mathrm{sec}$ ). Each of the 24 subjects was given a unique ordering of these four intensities, and eight cycles were administered, making 32 stimuli in all. In order to reduce the importance attributed by the subjects to these low-level stimuli, the tones were not mentioned. Instead, the experiment was presented as an investigation of the correlation of the physiological measures used over time, and the earphones were supposedly used to mask intermittent noises from the elevator servicing the building. A pilot study indicated that this device was successful, with subjects reporting that they could "still hear some elevator noises." It was thus expected that the stimuli used would be essentially neutral, with any effect attributable solely to their physical parameters.

For each stimulus presentation, and for each EEG channel, a measure of the change in activity in the alpha frequency range $(8-13 \mathrm{~Hz})$ was hand-scored off the record. This involved the use of a cursor constructed in the following manner: An isosceles triangle with sides $100 \mathrm{~mm}$ and base $5 \mathrm{~mm}$ was scribed on perspex. Lines were ruled $37.50 \mathrm{~mm}$ and $23.08 \mathrm{~mm}$ from the apex (measured along the sides) parallel to the base. These produced intercepts of $1.875 \mathrm{~mm}$ and $1.154 \mathrm{~mm}$, the wavelengths corresponding to 8 and $13 \mathrm{~Hz}$, respectively, at a paper speed of $15 \mathrm{~mm} / \mathrm{sec}$. This cursor was used to provide the frequency limits of EEG activity whose amplitudes were calculated as outlined below.

The activity level in each of the 1-sec intervals immediately before and after stimulus onset was obtained by summing successive vertical displacements of the pen tracing about the mean zero level for that 1-sec period. This measure of alpha activity in 1 -sec intervals has been used by Barry and Beh (1972) to characterize the EEG OR to visual stimulation, and by the same authors (1976) to investigate EEG events in relation to the visual afterimage. These two activity figures were combined to give the percentage change in alpha activity at stimulus onset.

\section{RESULTS}

The change in alpha activity in each channel is displayed as a function of repeated stimulus cycles in Figure 1 and as a function of stimulus intensity in Figure 2. The individual changes, which can be taken as the EEG ORs, were analyzed using a three-way repeated measures analysis of variance with stimulus repetition, stimulus intensity, and recording site as the three variables of interest. There was a significant repetition effect at the .001 level $[F(7,2185)=7.97$, $\left.M S_{e}=942.97\right]$, indicating habituation of the EEG response as required of an $\mathrm{OR}$ indicator. There was also a significant intensity effect $[F(3,2185)=8.56]$. 


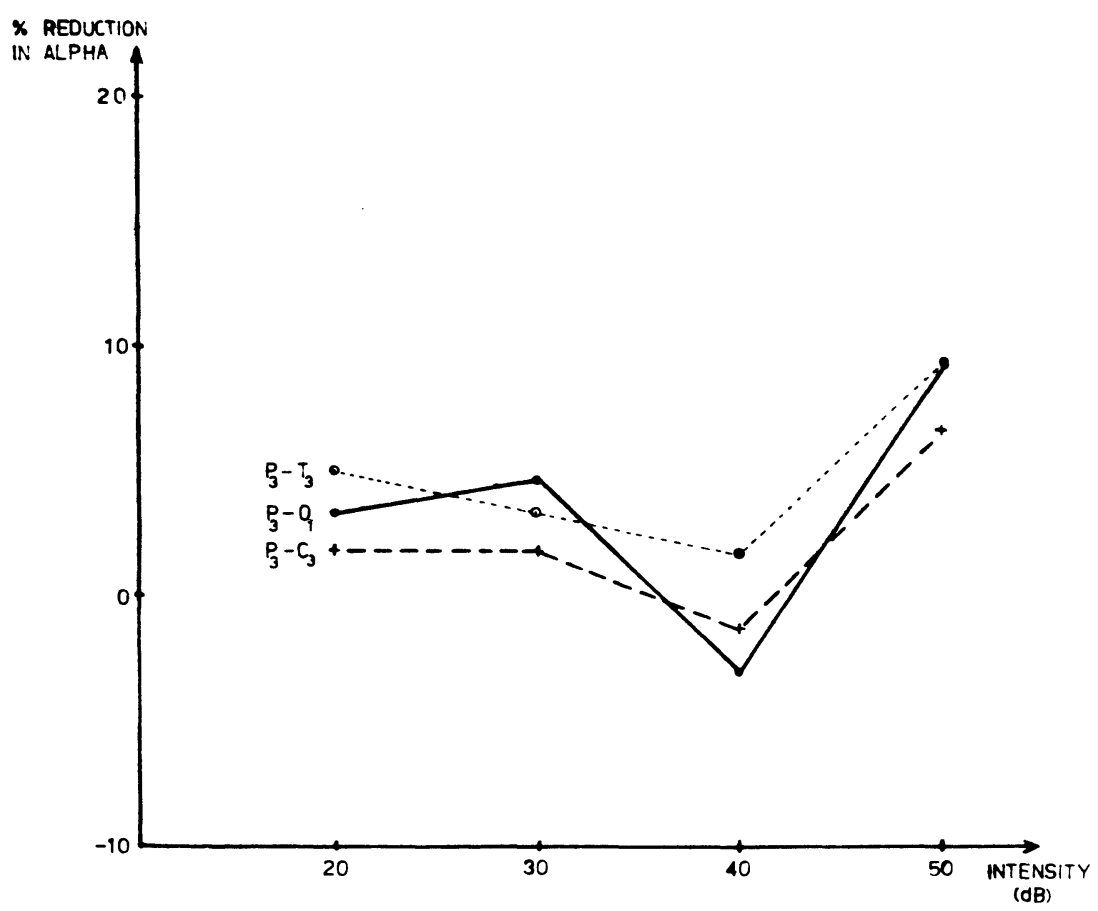

Figure 2. Change in alpha activity in each channel as a function of stimulus intensity.

There was no other significant main effect or interaction, indicating that there is no difference in the magnitude of the EEG ORs recorded from different sites, their dependence on stimulus intensity, or their habituation rates.

\section{DISCUSSION}

This study has specifically sought evidence for the local auditory EEG OR. Sokolov has widely claimed the existence of such a phenomenon characterized by a slower habituation of alpha desynchronization in the auditory projection area, compared with other cortical areas, when auditory stimulation is repeatedly presented. While a general habituation of the response measure used and its differential response to varying stimulus intensity was found (as predicted by Sokolov of any OR indicator), no such difference in habituation rate was obtained. This failure reinforces the lack of differential responding in the temporal and occipital areas to visual and auditory stimulation reported by Gastaut et al. (1957) and must cast considerable doubt on the validity of the local/general OR dichotomy as developed in Sokolov's theory.

It is important to emphasize that this is the first study to employ quantitative EEG measures in a statistical anal; sis of the local OR and that this points up some of the problems inherent in Sokolov's approach. The first of these concerns the use of an individual's data rather than that derived from group analysis. In the field of electroencephalography, there is sufficient variability in responsiveness to allow the finding of almost any response type in individual protocols, and it is possible that the changes in EEG responses found by Sokolov in individual recordings have been accorded undue generality and status. This possibility is supported by the lack of statistical analysis in the published work of Sokolov: we are shown illustrations of hypothetical processes, and in this particular area only individual protocols have been presented as illustrations.

In attempting to investigate this area of psychophysiology, it is apparent that the response measured should be identifiable as Sokolov's OR. In this study, two aspects of the procedure have been directed to such an end. The most important of these concerns the stimulus intensities used: low-level stimuli were used here to avoid the problems of defensive response (DR) elicitation. Unfortunately, low-level stimuli are associated with small response magnitudes, which, with the variability inherent in EEG responses, results in relatively obscure response trends. For example, it appears from Figures 1 and 2 that greater response magnitudes have been obtained in the auditory channel. Such a finding is supportive of the concept of an auditory analyzer, but has failed to reach statistical significance in this study. The second procedural aspect of relevance to Sokolov's methodology concerns the use of cycles of stimulus intensity. This procedure of allotting a unique stimulus cycle to each subject to eliminate the effect of intensity order follows a rare example provided by Sokolov (1963b). Together, these aspects of the procedure serve to allow an expectation that the "local" OR, if it exists, should have been found in this study. 
Such a failure to find evidence for the "local" EEG OR must add to the problems appearing in the literature with other aspects of the OR. Primarily, if the "local" OR has no validity, then all OR indicators should behave in a unitary fashion as regards habituation with stimulus repetition. To date, differences in habituation rates have been taken by Sokolov as reflecting the existence of a local/general dichotomy, with little specific investigation being carried out to support such a division. If this dichotomy fails to obtain further support in the experimental literature, then the problem of differences in habituation rates, reviewed recently by Graham (1973), would appear to be extremely embarrassing for Sokolov's OR concepts and the theory of attentive behavior built upon them.

\section{REFERENCES}

BARRY, R. J., \& BEH, H. C. EEG desynchronization as a function of visual stimulation. Psychonomic Science, 1972, 26, $241-242$.

BARRY, R. J., \& BEH, H. C. EEG correlates of the afterimage of visual stimulation. Psychophysiology, 1976, 13, 75-80.

Gastaut, H., Jus, A., Jus, C., Morrell, F., Storm van Leeuwen, W., Dongier, S., Naquet, R., Regis, H., Roger, A., Bexkering, D., Xamp, A., \& Werre, J. Etude topragraphique des réactions électroencephalographiques conditionnées chez l'homme. Electroencephalography and Clinical Neurophysiology, 1957, 9, 1-34.
Graham, F. K. Habituation and dishabituation of responses innervated by the autonomic nervous system. In $H$. V. S. \& M. J. Herz. Habituation. New York: Academic Press, 1973.

Grossman, S. P. A textbook of physiological psychology. New York: Wiley, 1967.

JASPER, H. H. Report of the committee on methods of clinical examination in electroencephalography. Electroencephalography and Clinical Neurophysiology, 1958, 10, 370-375.

LYNN, R. Attention, arousal and the orientation reaction. London: Pergamon Press, 1966.

Sharpless, S., \& Jasper, H. H. Habituation of the arousal reaction. Brain, 1956, 79, 655-680.

Sokolov, E. N. The higher nervous activity and the problem of perception. Acta Psychologia, 1955, 13, 134-135.

Soxolov, E. N. Neuronal models and the orienting reflex. In M. A. Brazier (Ed.), The central nerwous system and behavior: Transactions of the third conference. New York: Macy, 1960.

Sokolov, E. N. Effect of darkness on the human electroencephalogram (in Russian). Pavlov Journal of Higher Nervous Activity, 1961, 11, 411-418.

Sokolov, E. N. Higher nervous functions: The orienting reflex. Annual Reviews of Physiology, 1963, 25, 545-580. (a)

Sokolov, E. N. Perception and the conditioned reflex. Oxford: 1963. (b)

Sorolov, E. N. The modelling properties of the nervous system. In M. Cole \& I. Maltzman (Eds.), Handbook of contemporary Soviet psychology. New York: Basic Books, 1968.

(Received for publication July 21, 1975; accepted December 8, 1975.) 